\title{
THE POINT OF POINTLESS TOPOLOGY ${ }^{1}$
}

\author{
BY PETER T. JOHNSTONE
}

Introduction. A celebrated reviewer once described a certain paper (in a phrase which never actually saw publication in Mathematical Reviews) as being concerned with the study of "valueless measures on pointless spaces". This article contains nothing about measures, valueless or otherwise; but I hope that by giving a historical survey of the subject known as "pointless topology" (i.e. the study of topology where open-set lattices are taken as the primitive notion) I shall succeed in convincing the reader that it does after all have some point to it. However, it is curious that the point (as I see it) is one which has emerged only relatively recently, after a substantial period during which the theory of pointless spaces has been developed without any very definite goal in view. I am sure there is a moral here; but I am not sure whether it shows that "pointless" abstraction for its own sake is a good thing (because it might one day turn out to be useful) or a bad thing (because it tends to obscure whatever point there might be in a subject). That much I shall leave for the reader to decide.

This article is in the nature of a trailer for my book Stone spaces [35], and detailed proofs of (almost) all the results stated here will be found in the book (together with a much fuller bibliography than can be accommodated in this article). However, I should make it plain that I do not claim personal credit for more than a small proportion of these results, and that my own understanding of the nature of pointless topology has been enriched by my contacts with a number of other mathematicians, amongst whom I should particularly mention Bernhard Banaschewski, Michael Fourman, Martin Hyland, John Isbell, André Joyal and Myles Tierney. I should also mention the work of Bill Lawvere, particularly as reported in [41], on the nature of continuous variation and the conceptual relation between constant and variable quantities, which has had a profound influence on the developments which I wish to describe; but such questions as these will not be explicitly considered in the present article.

1. Lattices and spaces. It is well known that Hausdorff [21] was the first mathematician to take the notion of open set (or neighbourhood) as primitive in the study of continuity properties in abstract spaces. (As Fingerman [14] has

\footnotetext{
Received by the editors April 28, 1982.

1980 Mathematics Subject Classification. Primary 06A23, 18B30, 54A05; Secondary 01A60, $06 \mathrm{D} 05,18 \mathrm{~B} 25$.

${ }^{1}$ This article is based on a lecture given as part of the University of Chicago's "Friday Lecture Series" on 29th January 1982. The author is grateful to Saunders Mac Lane and Felix Browder for the suggestion that he write up the lecture in its present form.
}

(C)1983 American Mathematical Society 0273-0979/82/0000-0617/\$03.75 
pointed out, a measure of the "rightness" of Hausdorff's definition is the speed with which it, and Kuratowski's [39] equivalent definition in terms of a closure operation, supplanted all previous attempts - such as Fréchet's [17] - to define a notion of abstract space.) Thus from 1914 onwards it was known that a topological space was something which possessed a lattice of open subsets; but it was not until the middle thirties, with the work of Marshall Stone on the topological representation of Boolean algebras $[55,56]$ and distributive lattices [57], that this connection between topology and lattice theory began to be exploited.

Perhaps because of its elegant simplicity, which makes it appear today almost inevitable, the significance of Stone's representation theorem in the history of mathematics is often overlooked. Its influence (or lack of it) on the origins of category theory has been considered elsewhere [45]; but what concerns us here is the revolutionary idea that it is possible to construct topologically interesting spaces from purely algebraic data (such as a Boolean algebra). Previously, apart from a few trivial examples, all topological spaces considered by mathematicians had had some geometric content: one started with bits of Euclidean space (or generalizations thereof), and then patched them together or otherwise pulled them about. Stone's work showed that topology had not only these geometrical inputs but also an inescapable algebraic (i.e. lattice-theoretic) content; it followed that a better understanding of the lattice theory could lead to the solution of problems which had arisen from the geometrical side.

The first person (apart from Stone) to exploit this possibility of applying lattice theory to topology was Henry Wallman [59], in a celebrated paper in which he used lattice-theoretic ideas to construct what is now called the "Wallman compactification" of a $T_{1}$ topological space. A much more systematic study of the "algebra of topology" was undertaken a few years later by McKinsey and Tarksi [47, 48]; and mention should also be made of the pioneering work of Nöbeling [51] in writing the first textbook in which general topology was consistently studied from the lattice-theoretic viewpoint.

However, a fundamental change in outlook came in the late fifties, with the work of Charles Ehresmann [12] and his student Jean Bénabou [4] (and, at about the same time, with the Cambridge Ph.D. theses of Dona and Seymour Papert $[52,53])$. Hitherto, the lattice theory had been simply a means to an end; the ultimate goal was still the study of topological spaces in the sense in which Hausdorff had understood them. Ehresmann's insight, arising from his study of "topological" and "differentiable" categories (see [13]), was that a lattice with the right distributivity property deserved to be studied as a generalized topological space in its own right, irrespective of whether it was representable as the open-set lattice of an actual space, and irrespective of whether it came embedded in a given Boolean algebra of "all subsets of the space".

Subsequently, a good many results from topology were extended to these "generalized spaces" (notably in a long series of papers [5-9] by Hugh Dowker and Dona Papert Strauss). But it was not until around 1972 that the real point of the subject began to emerge, first in an important paper by John Isbell [25] 
and then even more clearly in the unpublished work of André Joyal. Isbell's point was that, in those instances where the category of "generalized spaces" behaved differently from that of traditional topological spaces, it was not always (as had hitherto been assumed) to the former's disadvantage: in particular products of "generalized spaces" tended to be better-behaved than Tychonoff products of spaces. Joval's point was perhaps a more subtle one; in order to explain it, we shall first have to develop a little of the theory of pointless topology.

2. Frames and locales. Let $X$ be a topological space, and write $\Omega(X)$ for the lattice of open subsets of $X$. As a lattice, $\Omega(X)$ is complete; the finite meet and arbitrary join operations coincide with the set-theoretic operations of intersection and union, since these preserve openness, but for the meet of an infinite family we have to take the interior of the intersection. Moreover, the infinite distributive law

$$
a \wedge \bigvee S=\bigvee\{a \wedge s \mid s \in S\}
$$

holds for all $a \in \Omega(X)$ and $S \subseteq \Omega(X)$, since it involves only operations which coincide with the set-theoretic ones. And if $f: X \rightarrow Y$ is a continuous map of spaces, the induced map $f^{-1}: \Omega(Y) \rightarrow \Omega(X)$ preserves finite meets and all joins, for essentially the same reason.

Thus we are interested in the category whose objects are complete lattices satisfying the distributive law (*), and whose morphisms are maps preserving finite meets and arbitrary joins. Ehresmann's name for such lattices was "local lattices"; but since the word "local" has other potential uses in lattice theory, we prefer the term frame, which was introduced by Dowker. However, there is one complication: the passage from $X$ to $\Omega(X)$, regarded as a functor from spaces to frames, is contravariant, and so our category of "generalized spaces" should be the opposite of the category of frames. It was first pointed out by Isbell that it is convenient to give a different name to the objects of this opposite category; we shall follow him in calling it the category of locales. Thus the words "frame" and "locale" are extremely synonymous as long as no reference (explicit or implicit) is made to morphisms; but, for example, "subframe" and "sublocale" mean entirely different things. (A sublocale is the same thing as a quotient frame.)

The category of locales, then, is the category in which we really want to work; but the category of frames is of some interest in it own right. In particular, it is an algebraic category (varietal, in the terminology of [43]); that is, the foregetful functor from frames to sets is monadic. What this means in practice is that we can define a frame by specifying generators and relations for it, in the same way that we are accustomed to specify presentations for groups and other familiar algebraic structures.

As an example of the use of this technique, let us consider the problem of defining the product of two locales. When we want to define the topological product of two spaces $X$ and $Y$, we start with the basic "open rectangles" $U \times V(U \in \Omega(X), V \in \Omega(Y))$ and then take the topology generated by this family of subsets of $X \times Y$. Now we can identify the open rectangles with 
elements of the Cartesian product ${ }^{2} \Omega(X) \times \Omega(Y)$; this suggests that, in order to define the locale product of two locales $A$ and $B$, we should take the elements of $A \times B$ as generators and then write down the relations that we expect to hold between open rectangles in a product space.

What relations should we expect to hold? First, the intersection of two open rectangles is an open rectangle; this gives us the family of relations

$$
\left(a_{1}, b_{1}\right) \wedge\left(a_{2}, b_{2}\right)=\left(a_{1} \wedge a_{2}, b_{1} \wedge b_{2}\right)
$$

for all $a_{1}, a_{2} \in A$ and $b_{1}, b_{2} \in B$. The union of two open rectangles is not in general a rectangle; but if we take a family of rectangles of the form $U_{\alpha} \times V$, where the second factor remains constant as $\alpha$ varies, their union is the rectangle $\left(\cup_{\alpha} U_{\alpha}\right) \times V$. This gives us the relations

$$
\bigvee\{(s, b) \mid s \in S\}=(\bigvee S, b)
$$

for all $S \subseteq A, b \in B$; and similarly

$$
\bigvee\{(a, t) \mid t \in T\}=(a, \vee T)
$$

for $a \in A$ and $T \subseteq B$. We now define $A \times{ }_{1} B$ to be the frame generated by the elements of $A \times B$ subject to these relations; of course, some work is needed to verify that $A \times{ }_{1} B$ is indeed the product of $A$ and $B$ in the category of locales (i.e. their coproduct in the category of frames), but the verification is straightforward. (Furthermore, it is now an easy exercise for anyone who knows the definition of the Tychonoff topology on an infinite product of spaces to extend the above description to construct infinite products of locales.)

If $A$ and $B$ happen to be open-set lattices $\Omega(X)$ and $\Omega(Y)$, then the identification of elements of $A \times B$ with open rectangles in $X \times Y$ gives rise to a frame map

$$
\Omega(X) \times{ }_{1} \Omega(Y) \rightarrow \Omega(X \times Y)
$$

which is surjective since the open rectangles generate $\Omega(X \times Y)$. In general, however, this map is not an isomorphism. It is an isomorphism if either $X$ or $Y$ is locally (quasi-)compact (see [26] for a more general form of this result), but if we take both $X$ and $Y$ to be the space $\mathbf{Q}$ of rationals (with the Euclidean topology), then it can be shown that the map is not injective. That is to say, there are certain additional relations that hold between open rectangles in $\mathbf{Q} \times \mathbf{Q}$, which are not consequences of the relations (1)-(3) which we imposed above.

Viewed correctly, this discrepancy between $\Omega(\mathbf{Q}) \times{ }_{1} \Omega(\mathbf{Q})$ and $\Omega(\mathbf{Q} \times \mathbf{Q})$ should be seen as a defect of the latter, not of the former. Whilst it is possible (see [35, II 2.14]) to give an explicit description of (at least some of) the extra relations which hold in $\Omega(\mathbf{Q} \times \mathbf{Q})$, all such relations seem to be inordinately complicated, and they appear accidental coincidences rather than expressions of any intuitive idea of how open rectangles ought to behave. That is, we should think of $\Omega(\mathbf{Q}) \times{ }_{1} \Omega(\mathbf{Q})$ as expressing what $\Omega(\mathbf{Q} \times \mathbf{Q})$ really ought to be,

\footnotetext{
${ }^{2} \mathrm{We}$ are cheating slightly here: any set-theorist will tell you that $\varnothing \times V_{1}$ is the same set as $\varnothing \times V_{2}$ (since both are empty) whereas $\left(\varnothing, V_{1}\right)$ and $\left(\varnothing, V_{2}\right)$ may be distinct as elements of $\Omega(X) \times \Omega(Y)$. But for our present purposes it doesn't matter.
} 
were it not for the unfortunate fact that we are obliged (if we stay within the category of spaces) to represent it as a lattice of subsets of $\mathbf{Q} \times \mathbf{Q}$. As we remarked earlier, this point of view was first expressed by Isbell [25]; the particular result which caused him to adopt it was the fact that the property of paracompactness (which, being an "open covering" property, is easily formulated for locales) is inherited by products in the category of locales-whereas it is well known that the corresponding result fails in the category of spaces. ${ }^{3}$

3. Points of locales. So far, we have not tackled the problem of determining whether a given locale is representable as an open-set lattice. Since a point of a space $X$ may be identified with a continuous map from the one-point space 1 to $X$, it is reasonable to define a point of a locale $A$ as a locale map from the two-element locale $2=\Omega(1)$ to $A$-or equivalently, a frame map $p: A \rightarrow 2$. Since $p$ is in particular a lattice homomorphism, the sets $p^{-1}(0)$ and $p^{-1}(1)$ are a prime ideal in $A$ and its complementary prime filter; and either of them suffices to determine $p$. Moreover, the fact that $p$ preserves all joins may be expressed either by the assertion that $p^{-1}(0)$ is a principal prime ideal (i.e. the ideal of all elements below some particular prime element of $A$ ) or by the assertion that $p^{-1}(1)$ is a completely prime filter, i.e. is inaccessible by arbitrary joins and not just finite ones.

If we write $\operatorname{pt}(A)$ for the set of all points of $A$, then there is a natural topology on $\operatorname{pt}(A)$, consisting of the sets

$$
\varphi(a)=\{p \in \operatorname{pt}(A) \mid p(a)=1\}
$$

for all $a \in A$. This makes pt into a functor from locales to spaces, and in fact it is right adjoint to the functor $\Omega$ - the counit of this adjunction, when viewed as a frame map $A \rightarrow \Omega(\operatorname{pt}(A))$, is simply the function $a \mapsto \varphi(a)$. From the definition it is clear that $\varphi$ is always surjective, but once again it need not be injective; if it is, then we say that the locale $A$ has enough points, or that it is spatial. Locales of the form $\Omega(X)$ are always spatial; for an arbitrary $A$, $\Omega(\operatorname{pt}(A))$ is the best approximation to $A$ by a spatial locale (i.e. it is the coreflection of $A$ in the subcategory of spatial locales).

Now there are a number of theorems available (see [1 and 23], for example) which say that every locale of a certain type (typically, every locale satisfying some compactness assumptions) is spatial-so that, from one point of view, when we work with locales of these types we are really doing nothing more than a disguised version of classical point-set topology. But one common feature of these theorems is that they all invoke nonconstructive principles such as the axiom of choice, and it is easy to see why this should be so: we have already observed that finding points of a given locale is tantamount to finding certain prime ideals, which is well known to be impossible without the aid of choice principles. (Of course, it is known [20] that the Prime Ideal

\footnotetext{
${ }^{3}$ The reader might be emboldened by this result to ask whether normality is inherited by locale products. Alas, the answer is no: for if $X$ is normal but not paracompact, then $X \times \beta X$ is not normal ([60, Theorem 21.1]), and (local) compactness of $\beta X$ ensures that this yields a counterexample for locales as well as spaces.
} 
Theorem for distributive lattices does not actually imply the axiom of choice; but the difference between them is insignificant compared with the difference between either and a constructive approach to mathematics.)

It is here that the real point of pointless topology begins to emerge; the difference between locales and spaces is one that we can (usually) afford to ignore if we are working in a "classical" universe with the axiom of choice available, but when (or if) we work in a context where choice principles are not allowed, then we have to take account of the difference-and usually it is locales, not spaces, which provide the right context in which to do topology. This is the point which, as I mentioned earlier, André Joyal began to hammer home in the early 1970s; I can well remember how, at the time, his insistence that locales were the real stuff of topology, and spaces were merely figments of the classical mathematician's imagination, seemed (to me, and I suspect to others) like unmotivated fanaticism. I have learned better since then.

Of course, a point as vague as this requires an illustration. For a start, let us consider the class of coherent locales: a locale $A$ is said to be coherent if it is isomorphic to the lattice $\operatorname{Idl}(B)$ of ideals of a distributive lattice $B$. It is easy to prove that $\operatorname{Idl}(B)$ is a frame whenever $B$ is a distributive lattice; in fact the assignment $B \mapsto \operatorname{Idl}(B)$ is a left adjoint to the forgetful functor from frames to distributive lattices. Moreover, we can recover $B$ from $\operatorname{Idl}(B)$ (up to isomorphism) as the sublattice of principal ideals; and the principal ideals can be characterized lattice-theoretically in $\operatorname{Idl}(B)$ as being exactly the finite (compact, or intranscessible, if you prefer) elements of this complete lattice. Thus we can characterize coherent locales in "topological" terms, by the behaviour of their compact elements, and each coherent locale determines a unique distributive lattice-in fact we have a duality between the category of distributive lattices and a category of coherent locales (though the latter does not admit arbitrary locale maps as morphisms). This algebraic triviality is the essence of the Stone representation theorem [57] for distributive lattices. ${ }^{4}$

To recover this representation in its usual form, we need two things: first, a theorem to the effect that every coherent locale is spatial, and then an identification of the spaces of points of coherent locales with the spectral spaces of Hochster [22] (i.e. the spaces which occur in Stone's representation theorem, as the spectra of commutative rings with 1 , and in all sorts of other representation theorems for different kinds of algebraic structures). Now the second of these is trivial, given Hochster's characterization of spectral spaces; so the key (nonconstructive) part of the representation theorem must be the first. But by the adjunction already mentioned, points of a coherent locale $\operatorname{Idl}(B)$ correspond to lattice homomorphisms $B \rightarrow 2$, and hence to prime ideals (or prime filters) of $B$; so the assertion "every coherent locale is spatial" is directly equivalent to the Prime Ideal Theorem for distributive lattices.

\footnotetext{
${ }^{4}$ To obtain Stone's earlier representation [55] for Boolean algebras, we simply have to observe that a distributive lattice $B$ is Boolean if and only if the locale $\operatorname{Idl}(B)$ is zero dimensional (i.e. generated by its complemented elements).
} 
4. Compact Hausdorff spaces. At this point, a topologist (echoing Kuyk [40, p. 164-168]) might say "Playing around with spectral spaces is all very well, but it isn't really topology; indeed, the representation theorems show that it is nothing more than algebra wearing a false beard. Real topologists concern themselves with spaces that satisfy the Hausdorff axiom (and are, in consequence, very rarely spectral); what, if anything, has the theory of locales to offer us?"

Perhaps our hypothetical topologist would claim to be interested in the category of compact Hausdorff spaces. If so, he would be well advised (though he may not believe it yet) to take an interest in a curious result which says that any compact Hausdorff space may be embedded as a retract of a spectral space. (The history of this result is rather obscure. It was in principle known to Wallman in 1938 [59], since it is an easy corollary of his construction of the Wallman compactification; but so far as I am aware it has not been explicitly stated anywhere until very recently.) Of course, from the point of view of our traditional topologist, the result is rather superfluous, since it seeks to represent something well known (compact Hausdorff spaces) in terms of something less well known (spectral spaces). But when we look at things from the localic viewpoint, they are definitely the other way around: as we have already seen, the class of coherent locales is extremely well behaved and much easier to work with than the class of "compact Hausdorff" locales.

Actually there is a small problem here. It is easy enough to see what it means for a locale to be compact, but the Hausdorff axiom for spaces is normally phrased in terms of points and so does not translate directly to locales. One way out of this difficulty would be to take as a definition the theorem that a space $X$ is Hausdorff if and only if the diagonal map $X \rightarrow X \times X$ is a closed inclusion; but since (as we observed earlier) locale products do not always coincide with space products, there is no reason to suppose that the corresponding condition on locales will be equivalent to the traditional one in the spatial case. (It turns out to be strictly stronger; see [25].) We therefore sidestep the problem by taking regularity, rather than the Hausdorff property, as our basic separation axiom for locales, and relying on the theorem that a (locally) compact Hausdorff space is regular.

How do we express regularity without mentioning points? One way to phrase the regularity axiom for spaces is to say that the closed neighbourhoods form a base at any point, or equivalently that any open set $U$ can be covered by open sets $V$ whose closures are contained in $U$. Accordingly, we define a locale to be regular if every $a \in A$ satisfies

$$
a=\vee\{b \in A \mid(\exists c \in A)(b \wedge c=0 \text { and } a \vee c=1)\}
$$

Then, as expected, we can prove that every compact regular locale $A$ can be expressed as a retract of a coherent locale $B$; in fact there is a canonical choice for $B$, namely $B=\operatorname{Idl}(A)$. (The result [32] actually does slightly better than this: it completely characterizes those locales which can occur as retracts of coherent locales. They turn out to be those locales which are compact and locally compact, and satisfy an additional "stability" property which in spatial 
terms means that the intersection of two compact subsets is compact. See also [54], for an investigation of the corresponding category of spaces.)

Given this result, it is easy to transfer known facts about coherent locales to the class of compact regular locales (or to the larger class described in the parenthesis above). For example, it is a triviality that the class of coherent locales, being the image of a left adjoint functor, is closed under frame coproducts (i.e. locale products); this transfers to yield a "Tychonoff theorem" for compact regular locales which is entirely constructive, whereas the Tychonoff theorem for compact Hausdorff spaces is known [44] to be equivalent to the Prime Ideal Theorem. (Actually, one can prove the Tychonoff theorem for arbitrary compact locales without using choice [33]; but the argument is not wholly constructive since it requires a transfinite induction.) Similarly, if one assumes the Prime Ideal Theorem, then the existence of enough points in coherent locales (already discussed) yields the existence of enough points in compact regular locales, and hence an equivalence of categories between compact regular locales and compact Hausdorff spaces.

Point-set topology abounds in theorems and constructions involving compact Hausdorff spaces which require the axiom of choice, or at least the Prime Ideal Theorem-and which, in many cases, can be shown to be logically equivalent to the latter. In nearly every case, it turns out that the corresponding theorems and constructions for locales can be carried out constructively (and often with fewer topological restrictions on the initial data). Thus we see that the only nonconstructive element in the original topological theorems was the insistence on identifying compact regular locales with their spaces of points.

To give a few examples: first, there is a constructive "Stone-Čech compactification" for locales [3], which specializes in the presence of the Prime Ideal Theorem to the usual one for spaces. The minimal projective cover of a space, first constructed by Gleason [18] for (locally) compact Hausdorff spaces and subsequently extended by other authors to more general classes of spaces, requires the Prime Ideal Theorem since it is defined as a space of prime filters; but it has a localic version [31] which works without any such assistance (and which, moreover, is as simple to construct for arbitrary locales as for compact regular ones, in constrast to what happens for spaces). Classically, the hyperspace of a compact Hausdorff space (i.e. the space of closed subsets, with the Vietoris topology [58]) can be proved compact only if one assumes the Prime Ideal Theorem; but from the localic viewpoint it is part of a construction definable on arbitrary locales [36], which has all the formal properties of the Vietoris hyperspace, and which inherits compactness and regularity from the locale which generates it.

In each of the above examples, the idea which leads to a constructive proof is essentially the same, and is that which we already used in constructing locale products: we start with an intuitive idea of what the (sub)basic open sets ought to be, and of what relations ought to hold between them, and then we appeal to the algebraicity of the category of frames in order to take these intuitive ideas as a definition. Provided we don't impose the additional restriction that the locale we're constructing should be representable as an open-set lattice, it 
then becomes a matter of mere algebra (admittedly, infinitary algebra, but that is not something to be frightened of) to verify that it has the formal properties we want. (Another example of the same technique, exploited in [24], is the construction of "function-spaces" $B^{A}$ (i.e. exponentials in the category of locales) where $A$ is locally compact and $B$ is any locale; but it doesn't quite belong with those mentioned above because the corresponding construction on spaces does not require any use of choice principles. There is also a rather different construction of function-locales, sketched in [50].)

5. The point of constructive topology. A classical topologist may be forgiven for feeling that we have still not arrived at the point. The fact that classical theorems of topology have constructive proofs in the localic context is undoubtedly pretty, but it isn't obviously useful to a man who is happy to assume the axiom of choice whenever he needs it in his work. To such a man, it may appear that we are doing "topology for logicians" rather than "topology for topologists".

Nevertheless, even the most severely pragmatic of topologists can gain something by learning to do topology constructively. The point is that there are, even within the universe of classical mathematics, contexts in which one would like to do topology but one doesn't have available the law of excluded middle or the axiom of choice. Such contexts are called toposes; for the purposes of the present article, it isn't necessary to know exactly what a topos is (but see [29] for a detailed account of the subject, or [46] for a more introductory one), but only that a topos is a category which is sufficiently like the category of sets for one to carry out set-theoretic constructions inside it, and that (provided one doesn't insist on the law of excluded middle being valid) examples of toposes abound in mathematics, and are such that mathematics done "internally" in them often has an interesting "external" interpretation.

For instance, given any space $X$ (or more generally any locale), the category of sheaves (of sets) over $X$ is a topos $\operatorname{Sh}(X)$, and topology done internally in this topos interprets as "topology over the fixed base $X$ ". Thus given any continuous map $f: Y \rightarrow X$, there is an internal locale in $\operatorname{Sh}(X)$ which completely represents it; more generally, we can represent any locale map $f$ : $B \rightarrow \Omega(X)$ in this way, but even if $B$ has enough points (i.e. $B \cong \Omega(Y)$ for some $Y$ ), there is no guarantee that the internal locale which represents it will also be spatial. (Explicitly, internal spaces in $\operatorname{Sh}(X)$ correspond to continuous maps $f: Y \rightarrow X$ which have "enough local sections", in the sense that every point of $Y$ is in the image of a continuous section of $f$ over an open subset of $X$.

In this context, topological properties of internal locales interpret externally as familiar properties of continuous maps. For example, compact regular locales in $\operatorname{Sh}(X)$ correspond almost exactly to proper maps $f: Y \rightarrow X$ ("almost" because in one direction the implication requires mild separation assumptions on $X$ and/or $Y$; for a precise statement of the theorem, see [32]); this gives a mathematical formulation of the intuitive idea, which has surely been present since proper maps were first studied [42], that a proper map is a "relativized" 
compact Hausdorff space. (Similarly, zero-dimensional locales in $\operatorname{Sh}(X)$ correspond to spreads in the sense of [16], open maps of spaces have a topostheoretic interpretation [30], ${ }^{5}$ and so on.) Thus the results about compact regular locales which we mentioned earlier, because their proofs are constructively valid, may be interpreted in toposes of the form $\operatorname{Sh}(X)$ to yield theorems about proper maps. As an example, the Stone-Čech compactification of locales yields a factorization theorem, originally due to Dyckhoff [10], which factors an arbitrary map $Y \rightarrow X$ in the best possible way through a proper map $Z \rightarrow X$; see [34] for the details.

Another context in which the internal theory of locales promises to yield interesting external theorems is that of equivariant topology. If $G$ is a group (or more generally a monoid), the category of $G$-sets is a topos, and internal locales in this topos correspond to external locales equipped with a $G$-action. Actually, if $G$ is a (discrete) group, the topos of $G$-sets is sufficiently like the category of sets (in particular, it retains enough of the axiom of choice) to enable us to work with internal spaces rather than locales if we prefer; but if we wish (as in [2]) to study actions of monoids which are not groups, and still more if we wish to study continuous actions of topological groups, then we are forced once again to work with locales.

So far, relatively little work has been done on specific applications of locale theory in contexts like these; so it is perhaps appropriate to conclude this article by mentioning some areas which (in the writer's opinion, at least) seem ripe for study in this way. One is homotopy theory: the work of Joyal, Fourman and Hyland [15] shows that in a constructive context it may be necessary to regard the real line as a (nonspatial) locale, at least if we wish to retain the Heine-Borel theorem that its closed bounded subsets are compact. So there is scope for developing the basic ideas of homotopy theory for locales, starting from the localic notion of the unit interval; when interpreted in the two contexts mentioned above, it should yield results in the "Ex-homotopy theory" and "equivariant homotopy theory" that have been studied in recent years by James [27, 28].

Another area of topology which cries out to be developed for locales is dimension theory. Much work has been done by dimension-theorists (see [49], for example) on dimension-raising and dimension-lowering maps $f: Y \rightarrow X$; it seems clear that there should be a link between these notions and some internal notion of dimensionality in $\operatorname{Sh}(X)$. The work of Dyckhoff [11] and Zarelua [63] may well provide pointers to the correct way of approaching this subject, although they concern themselves largely with abelian sheaves rather than sheaves of sets.

\footnotetext{
${ }^{5}$ A locale $A$ is said to be open $[19,37]$ if there is a map from $A$ to the set of truth-values which associates to each $a \in A$ "the extent to which $a$ is inhabited (i.e. nonempty)". Classically, such a map trivially always exists (it sends 0 to 0 and everything else to 1); this corresponds to the fact that every continuous map into a one-point space is open. The work of Kirwan [38] suggests that openness should be an important property in the constructive theory of uniform locales (i.e. the localic analogue of uniform spaces).
} 
Finally, as already hinted in a footnote, it could well be profitable to develop the theory of uniform locales. In [25], Isbell did some work on uniform locales from a classical viewpoint, and showed that they were indeed of considerable interest, but no one has yet produced a constructive development of the theory. Assuming that such a development is possible (and there do seem to be some nontrivial obstacles in the way of it), the resulting theorems would surely have worthwhile applications in $\operatorname{Sh}(X)$ and other toposes; they should also help to clarify the subject of localic groups, in which interesting work has already been done by Wraith [61, 62].

\section{REFERENCES}

1. B. Banaschewski, The duality of distributive continuous lattices, Canad. J. Math. 32 (1980), 385-394.

2. Extension of invariant linear functionals: Hahn-Banach in the topos of M-sets, J. Pure Appl. Algebra 17 (1980), 227-248.

3. B. Banaschewski and C. J. Mulvey, Stone-Čech compactification of locales, I, Houston J. Math. 6 (1980), 301-312.

4. J. Bénabou, Treillis locaux et paratopologies, Séminaire Ehresmann (Topologie et Géométrie Différentielle), 1 re année (1957-58), exposé 2.

5. C. H. Dowker and D. Papert, Quotient frames and subspaces, Proc. London Math. Soc. 16 (1966), 275-296.

6. On Urysohn's lemma, General Topology and its Relations to Modern Analysis and Algebra (Proc. Prague Symposium, 1966), Academia, Prague, 1967, pp. 111-114.

7. C. H. Dowker and D. Strauss, Separation axioms for frames, Colloq. Math. Soc. János Bolyai 8 (1972), 223-240.

8. __ Paracompact frames and closed maps, Sympos. Math. 16 (1975), 93-116.

9. __ Sums in the category of frames, Houston J. Math. 3 (1977), 7-15.

10. R. Dyckhoff, Factorization theorems and projective spaces in topology, Math. Z. 127 (1972), 256-264.

11. Categorical methods in dimension theory, Categorical Topology (Proc. Mannheim Conf., 1975), Lecture Notes in Math., vol. 540, Springer-Verlag, Berlin and New York, 1976, pp. 220-242.

12. C. Ehresmann, Gattungen von lokalen Strukturen, Jber. Deutsch. Math.-Verein. 60 (1957), $59-77$

13. Catégories topologiques et catégories différentiables, Colloq. Géom. Diff. Globale (Bruxelles), Centre Belge Rech. Math., Louvain, 1959, pp. 137-150.

14. J. Fingerman, The historical and philosophical significance of the emergence of point-set topology, Ph.D. thesis, Univ. of Chicago, 1981.

15. M. P. Fourman and J. M. E. Hyland, Sheaf models for analysis, Applications of Sheaves (Proc. Durham Symposium, 1977), Lecture Notes in Math., vol. 753, Springer-Verlag, Berlin and New York, 1979, pp. 280-301.

16. R. H. Fox, Covering spaces with singularities, Algebraic Topology (Sympos. in honor of S. Lefschetz), Princeton Univ. Press, Princeton, N. J., 1957, pp. 243-257.

17. M. Fréchet, Sur quelques points du calcul fonctionnel, Rend. Circ. Mat. Palermo 22 (1906), $1-71$.

18. A. M. Gleason, Projective topological spaces, Illinois J. Math. 2 (1958), 482-489.

19. R. J. Grayson, Constructive properties of complete Heyting algebras and related structures, preprint (1982).

20. J. D. Halpern, The independence of the axiom of choice from the Boolean prime ideal theorem, Fund. Math. 55 (1964), 57-66.

21. F. Hausdorff, Grundzüge der Mengenlehre, Veit \& Co., Leipzig, 1914.

22. M. Hochster, Prime ideal structure in commutative rings, Trans. Amer. Math. Soc. 142 (1969), $43-60$. 
23. K. H. Hofmann and J. D. Lawson, The spectral theory of distributive continuous lattices, Trans. Amer. Math. Soc. 246 (1978), 285-310.

24. J. M. E. Hyland, Function-spaces in the category of locales, Continuous Lattices (Proc. Bremen workshop, 1979), Lecture Notes in Math., vol. 871, Springer-Verlag, Berlin and New York, 1981, pp. 264-281.

25. J. R. Isbell, Atomless parts of spaces, Math. Scand. 31 (1972), 5-32.

26. __ Product spaces in locales, Proc. Amer. Math. Soc. 81 (1981), 116-118.

27. I. M. James, Ex-homotopy theory. I, Illinois J. Math. 15 (1971), 324-337.

28. __ Alternative homotopy theories, Enseign. Math. (2) 23 (1977), 221-237.

29. P. T. Johnstone, Topos theory, L.M.S. Math. Mono., no. 10, Academic Press, London, 1977.

30. __ Open maps of toposes, Manuscripta Math. 31 (1980), 217-247.

31. __ The Gleason cover of a topos. I, J. Pure Appl. Algebra 19 (1980), 171-192.

32. __ The Gleason cover of a topos. II, J. Pure Appl. Algebra 22 (1981), 229-247.

33. __ Tychonoff's theorem without the axiom of choice, Fund. Math. 113 (1981), 21-35.

34. __ Factorization theorems for geometric morphisms. II, Categorical Aspects of Topology and Analysis (Proc. Carleton Conf., 1980), Lecture Notes in Math., vol. 915, Springer-Verlag, Berlin and New York, 1982, pp. 216-233.

35. __ Stone spaces, Cambridge Univ. Press, Cambridge, 1982.

36. __ The Vietoris monad on the category of locales (in preparation).

37. A. Joyal and M. Tierney, An extension of the Galois theory of Grothendieck (to appear).

38. F. C. Kirwan, Uniform locales, Part III dissertation, Univ. of Cambridge, 1981.

39. K. Kuratowski, Sur l'opération $\bar{A}$ de l'analysis situs, Fund. Math. 3 (1922), 182-199.

40. W. Kuyk, Complementarity in mathematics, Math. and its applications, vol. 1, Reidel, Dordrecht, 1977.

41. F. W. Lawvere, Continuously variable sets: Algebraic geometry = geometric logic (Logic Colloq., 1973), Studies in Logic and the Foundations of Math., vol. 80, North-Holland, Amsterdam, 1975, pp. 135-156.

42. J. Leray, L'anneau spectral et l'anneau filtré d'homologie d'un espace localement compact et d'une application continue, J. Math. Pures Appl. 29 (1950), 1-80.

43. F. E. J. Linton, Some aspects of equational categories, Categorical Algebra (Proc. La Jolla Conf., 1965), Springer-Verlag, Berlin and New York, 1966, pp. 84-94.

44. J. koš and C. Ryll-Nardzewski, On the application of Tychonoff's theorem in mathematical proofs, Fund. Math. 38 (1951), 233-237.

45. S. Mac Lane, The influence of $M$. H. Stone on the origins of category theory, Functional Analysis and Related Fields, Springer-Verlag, Berlin and New York, 1970, pp. 228-241.

46. __ Sets, topoi and internal logic in categories (Logic Colloq., 1973), Studies in Logic and the Foundations of Math., vol. 80, North-Holland, Amsterdam, 1975, pp. 119-134.

47. J. C. C. McKinsey and A. Tarski, The algebra of topology, Ann. of Math. (2) 45 (1944), $141-191$.

48. __ On closed elements in closure algebras, Ann. of Math. (2) 47 (1946), 122-162.

49. J. Nagata, Modern dimension theory, Noordhoff, Groningen, 1965.

50. S. B. Niefield, Exactness and projectivity, Category Theory (Proc. Gummersbach Conf., 1981), Lecture Notes in Math., Springer-Verlag, Berlin and New York. (to appear).

51. G. Nöbeling, Grundlagen der analytischen Topologie, Die Grundlehren der math. Wissenschaften, Band 72, Springer-Verlag, Berlin and New York, 1954.

52. D. Papert, Lattices of functions, measures and point-sets, Ph.D. thesis, Univ. of Cambridge, 1958.

53. S. Papert, The lattices of logic and topology, Ph.D. thesis, Univ. of Cambridge, 1959.

54. H. Simmons, A couple of triples, Topology Appl. 13 (1982), 201-223.

55. M. H. Stone, Boolean algebras and their applications to topology, Proc. Nat. Acad. Sci. U.S.A. 20 (1934), 197-202.

56. The theory of representations for Boolean algebras, Trans. Amer. Math. Soc. 40 (1936), 37-111.

57. mat. fys. 67 (1937), 1-25.

58. L. Vietoris, Bereiche zweiter Ordnung, Monatsh. für Math. und Physik 32 (1922), 258-280. 
59. H. Wallman, Lattices and topological spaces, Ann. of Math. (2) 39 (1938), 112-126.

60. S. Willard, General topology, Addison-Wesley, Reading, Mass., 1970.

61. G. C. Wraith, Galois theory in a topos, J. Pure Appl. Algebra 19 (1980), 401-410.

62. __ Localic groups, Cahiers Topologie Géom. Différentielle 22 (1981), 61-66.

63. A. V. Zarelua, Sheaf theory and zero-dimensional mappings, Applications of Sheaves (Proc. Durham Sympos., 1977), Lecture Notes in Math., vol. 753, Springer-Verlag, Berlin and New York, 1979, pp. 768-779.

Department of Pure Mathematics, University of Cambridge, 16 Mill lane, Cambridge CB2 1SB, ENGLAND 
